POS $\quad$ PROCEEDINGS

\title{
Blazar origin of some IceCube events
}

\section{Sarira Sahu}

Instituto de Ciencias Nucleares, Universidad Nacional Autónoma de México, Circuito Exterior, C.U., A. Postal 70-543, 04510 México DF, México.

Astrophysical Big Bang Laboratory, RIKEN, Hirosawa, Wako, Saitama 351-0198, Japan

E-mail: sarira@nucleares.unam.mx

\section{Luis Salvador Miranda}

Department of Physics, University of Johannesburg, P.O. Box 524, Auckland Park 2006, South Africa

E-mail: smiranda-palacios@uj.ac.za

\section{Alberto Rosales de León*}

Instituto de Ciencias Nucleares, Universidad Nacional Autónoma de México, Circuito Exterior, C.U., A. Postal 70-543, 04510 México DF, México

E-mail: albertoros4@ciencias.unam.mx

In 2015 the ANTARES collaboration presented a time dependent analysis of a selected number of flaring blazars to look for upward going muon events produced from the charge current interaction of the muon neutrinos. We use the same list of flaring blazars to look for a possible positional correlation with the IceCube neutrino events. In the context of the photohadronic model we propose that the neutrinos are produced within the nuclear region of the blazar where Fermi accelerated high energy protons interact with the background synchrotron/SSC photons. Although we found that some objects from the ANTARES list are within the error circles of a few IceCube events, the statistical analysis shows that none of these sources have a significant correlation.

XVII International Workshop on Neutrino Telescopes

13-17 March 2017

Venezia, Italy

\footnotetext{
*Speaker.
} 


\section{Introduction}

Interactions of ultra high energy cosmic rays (UHECRs) with the background photons and protons produce high energy $\gamma$-rays and neutrinos. The IceCube detector located at South Pole in Antarctic ice is built precisely to look for high energy neutrinos (above few $\mathrm{TeV}$ ) by measuring the Cherenkov radiation of the secondary charged particles created in each neutrino event. In 2014 the IceCube collaboration published an analysis of 3 years of data (2010-2013) in which a total of 37 events were observed during 988-days [1]. These events have flavors, directions and energies inconsistent with those expected from the atmospheric muon and neutrino backgrounds.

There exist different types of potential astrophysical sources to produce UHECRs, high energy neutrinos and $\gamma$-rays. The list includes: Gamma-ray bursts (GRBs), core of active galactic nuclei (AGN), BL Lacerate objects (BL Lacs), flat spectrum radio quasars (FSRQ), starburst galaxies, among others. There are also nonstandard physics interpretations from the decay of superheavy dark matter particles, leptoquark interaction and decay of exotic neutrinos, etc.

In 2015 ANTARES collaboration presented a time dependent analysis to look for upward going muon tracks from flaring blazars, the collaboration selected 41 very bright and variable Fermi-LAT blazars with significant time variability and a flux $>10^{-9}$ photons $\mathrm{cm}^{-2} \mathrm{~s}^{-1}$ for the $\gamma$-ray energy above $1 \mathrm{GeV}$ [2]. In this work we analyzed a list of 41 Fermi-LAT flaring blazars to see if there is any correlation with the IceCube neutrino events.

\section{Flaring model of a blazar}

Blazars are believed to be likely candidates to produce UHECRs and neutrinos $[3,4,5]$. These extragalactic objects are characterized by relativistic jets with a small viewing angle with respect to the line of sight and are powered by a supermassive black hole in the centre. In this environment protons can reach ultra high energies through shock or diffusive Fermi acceleration processes with a power-law spectrum given as $d N / d E \propto E^{-\kappa}$, with the power index $\kappa \geq 2$ [6]. In the photohadronic scenario considered by Sahu et al. $[5,7,8]$ it is proposed that a high energy proton can interact with the background synchrotron/SSC photons to produce high energy $\gamma$-rays and neutrinos

$$
p+\gamma \rightarrow \Delta^{+}\left\{\begin{array}{c}
p \pi^{0} \\
n \pi^{+} \rightarrow n e^{+} v_{e} v_{\mu} \overline{v_{\mu}}
\end{array} .\right.
$$

In this model it is assumed that the flaring occurs within a compact and confined region with a comoving radius $R_{f}^{\prime}$ inside the blob of radius $R_{b}^{\prime}$, here the ' notation implies jet comoving frame. Both the internal and the external jets are moving with almost the same bulk Lorentz factor $\Gamma$ and the Doppler factor $\mathscr{D}$ (for blazars $\Gamma \simeq \mathscr{D}$ ). In the inner region, the photon density $n_{\gamma, f}^{\prime}$ is very high compared to the photon density $n_{\gamma}^{\prime}$ in the outer region i.e. $n_{\gamma, f}^{\prime} \gg n_{\gamma}^{\prime}$.

In the photohadronic interaction, the $\Delta$-resonance produced will give both high energy neutrinos and $\gamma$-rays, the relation between the seed photon and the neutrino energy is given by

$$
E_{v} \varepsilon_{\gamma}=0.016 \frac{\mathscr{D}^{2}}{(1+z)^{2}} \mathrm{GeV}^{2},
$$

where $E_{v}$ and $\varepsilon_{\gamma}$ are respectively the observed neutrino energy and the background photon energy. The source is located at a redshift $z$ and the bulk Lorentz factor of the jet is $\Gamma \simeq \mathscr{D}$. 


\section{Statistical analysis of the possible sources}

To identify the possible sources we employed the Unbinned Maximum Likelihood Method (UMLM) to look for a correlation between the blazar sample and the IceCube events. The signal and the background weights are not separable for an object, both contribute to the likelihood function, which is given by the product of the individual probability densities for the IceCube events [9]

$$
\mathscr{L}\left(n_{s}, \vec{x}_{s}\right)=\prod_{i=1}^{N}\left[\frac{n_{s}}{N} S_{i}\left(\vec{x}_{s}\right)+\left(1-\frac{n_{s}}{N}\right) B_{i}\right],
$$

where $\mathrm{N}$ is the number of IceCube events we take into account, $n_{s} / N$ is the weight associate with the signal probability density function (PDF) and its values vary between 0 and 1 .

The background PDF $B_{i}=\mathscr{B}\left(E_{i}, \delta_{i}\right)$ is constructed from the integrated effective areas of the IceCube 79 string configuration [10] and it takes into account the contribution from the atmospheric muon neutrinos. Above $\sim 100 \mathrm{TeV}$, neutrinos from the decay of charm hadrons $D^{ \pm}, D^{0}$ contribute to the background neutrino flux, this prompt flux is also considered [11]. The signal PDF is defined as a product of a spatial term and an energy term

$$
S_{i}=\mathscr{S}_{i}\left(\left|\mathbf{x}_{i}-\mathbf{x}_{s}\right|, \sigma_{i}\right) \mathscr{E}_{i}\left(E_{i}, \delta_{i}, \kappa\right),
$$

where

$$
\mathscr{S}_{i}\left(\vec{x}_{s}\right)=\frac{1}{2 \pi \sigma_{i}^{2}} e^{-\frac{\left|x_{i}-x_{s}\right|^{2}}{2 \sigma_{i}^{2}}} .
$$

In the above equation $\left|x_{i}-x_{s}\right|^{2}$ is the space angle difference between the source and the reconstructed event direction and $\sigma_{i}$ is the standard deviation of the $i^{t h}$ IceCube angular error distribution. The signal energy PDF $\mathscr{E}_{i}$ depends on the event energy, spectral index $\kappa$ and the declination.

The observed IceCube events can be modeled by taking into account two hypothesis: (1) the events could be produced by atmospheric muons and the muon neutrinos (background), or (2) from an astrophysical source which also includes the background contribution. A good test of compatibility is the ratio of these two hypothesis, the background of unique weight $\left(n_{s}=0\right)$ and the maximized likelihood of the second hypothesis with the corresponding $n_{s}$ values defined as $n_{s}=n_{s}^{*}$. To evaluate each point source we define a Test Statistic (TS) as minus twice the log of the likelihood ratio

$$
T S=-2 \log \left[\frac{\mathscr{L}\left(n_{s}=0\right)}{\mathscr{L}\left(n_{s}=n_{s}^{*}\right)}\right] .
$$

The compatibility of the second hypothesis depends on the estimate of the posteriori p-value. If the posteriori p-value is close to unity then it is consistent with the background.

\section{Results}

We calculate the significance of each source location running 10,000 simulations in which the right ascension of each IceCube sample event is randomized. The p-value is calculated as the number of simulations with $T S_{(s i m)} \geq T S$ divided by the total number of simulations for a given source, where $T S_{(s i m)}$ is the TS value obtained from a simulation. The posteriori p-value for each 


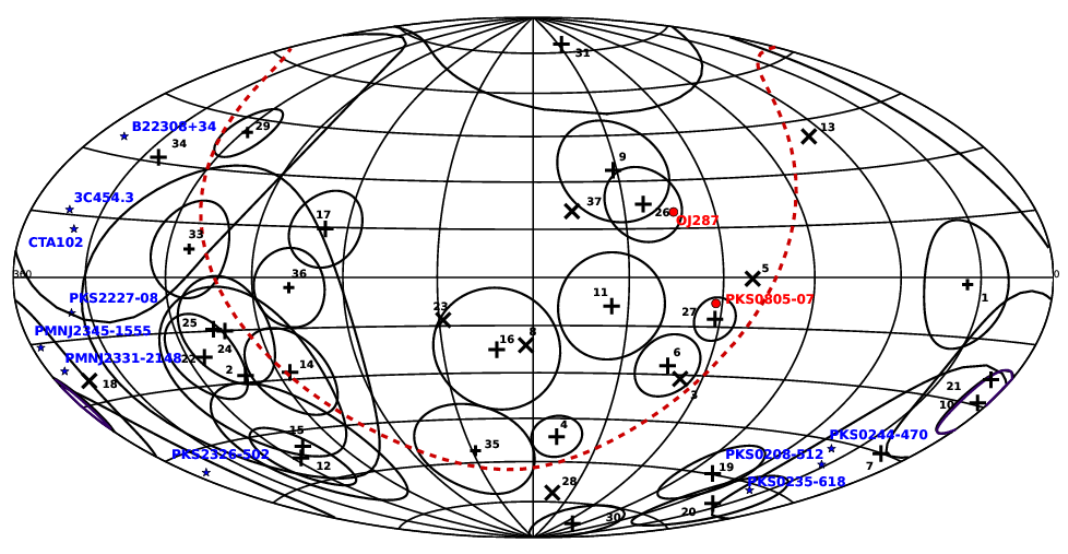

Figure 1: Sky map in Equatorial coordinates with the 37 IceCube events and their individual errors. + SIGN corresponds to shower event and $\times$ sign corresponds to track event. The objects of Table 1 are also shown, FSRQs in blue and BL Lac objects in red colors.

\begin{tabular}{lccccccc}
\hline Object & Type & (RA, Dec.) & $\begin{array}{c}\text { event } \\
\text { ID }\end{array}$ & $n_{s}^{*}$ & TS & $\begin{array}{c}\text { p } \\
\text { value }\end{array}$ & $\begin{array}{c}\text { post-p } \\
\text { value }\end{array}$ \\
\hline PKS2326-502 & FSRQ & $(352.32,-49.94)$ & 7 & 0.06 & 0.0008 & 0.44 & 1.0 \\
\hline PKS0208-512 & FSRQ & $(32.7,-51.2)$ & 7 & 0.59 & 0.109 & 0.22 & 1.0 \\
\hline PKS0235-618 & FSRQ & $(39.29,-61.62)$ & 7,20 & 0.39 & 0.040 & 0.18 & 1.0 \\
\hline PMNJ2345-1555 & FSRQ & $(356.27,-15.89)$ & 21 & 0.71 & 0.197 & 0.43 & 1.0 \\
\hline B22308+34 & FSRQ & $(347.77,34.43)$ & 34 & 0.97 & 0.503 & 0.48 & 1.0 \\
\hline PKS0244-470 & FSRQ & $(41.06,-47.06)$ & 7 & 0.73 & 0.179 & 0.17 & 1.0 \\
\hline CTA102 & FSRQ & $(338.12,11.72)$ & 34 & 0.77 & 0.249 & 0.53 & 1.0 \\
\hline PMNJ2331-2148 & FSRQ & $(352.75,-21.74)$ & 21 & 0.6 & 0.125 & 0.45 & 1.0 \\
\hline PKS2227-08 & FSRQ & $(337.44,-8.55)$ & 34 & 0.53 & 0.096 & 0.53 & 1.0 \\
\hline OJ287 & BL Lac & $(133.85,20.09)$ & 26 & 1.32 & 0.691 & 0.31 & 0.99 \\
& & & & 0.69 & 0.184 & 0.33 & 1.0 \\
\hline PKS0805-07 & BL Lac & $(122.06,-7.85)$ & 27 & 1.23 & 0.556 & 0.24 & 1.0 \\
& & & & 0.54 & 0.102 & 0.27 & 1.0 \\
\hline 3C454.3 & FSRQ & $(343.5,16.15)$ & 34 & 0.85 & 0.33 & 0.50 & 1.0 \\
& & & & 0.24 & 0.022 & 0.49 & 1.0 \\
\hline \hline
\end{tabular}

Table 1: The possible sources within the median angular error of an IceCube event are given here. The numerical results of the parameters from the statistical analysis are shown. 
object is estimated as the fraction of the randomized simulations that yields an equal or higher TS value for at least one of the 41 ANTARES sources.

From the 41 objects considered, 32 obtained a TS $>0$ for the spectral index $\kappa=2,12$ of these were within the median angular error of at least one IceCube event (Table 1). By including the prompt contribution to the background we found that 19 sources have TS $>0$, but only three objects (OJ287, PKS0805-07 and 3C454.3) inside the median angular error of three IceCube events $(26,27,34)$. These objects are listed in the bottom of the Table 1.The posteriori p-value of all the possible sources are $\geq 99 \%$. This shows that our results are consistent with the background fluctuation.

\section{Conclusions}

For our analysis we take into account the energy dependence, a spectral index $\kappa$ and the prompt contribution to the atmospheric flux, still there is not enough statistical evidence to claim a correlation of the 12 sources shown in the sky map (Fig. 4). It is possible that the individual high energy neutrino flux from these objects is below the IceCube limit. We have to wait for more data to look for a possible correlation of flaring Blazars with the IceCube events.

Acknowledgments. We thank S. Mohanty for many useful comments and discussions. The work of S. S. is partially supported by DGAPA-UNAM (Mexico) Projects No. IN110815.

\section{References}

[1] M. G. Aartsen et al. [IceCube Collaboration], Observation of High-Energy Astrophysical Neutrinos in Three Years of IceCube Data, Phys. Rev. Lett. 113, 101101 (2014).

[2] S. Adrian-Martinez et al. [ANTARES Collaboration], Search for muon-neutrino emission from GeV and TeV gamma-ray flaring blazars using five years of data of the ANTARES telescope, JCAP 1512, no. 12, 014 (2015).

[3] P. Padovani and E. Resconi, Are both BL Lacs and pulsar wind nebulae the astrophysical counterparts of IceCube neutrino events?, Mon. Not. Roy. Astron. Soc. 443, 474 (2014).

[4] F. Krauß et al., TANAMI Blazars in the IceCube PeV Neutrino Fields, Astron. Astrophys 566, L7 (2014).

[5] S. Sahu and L. S. Miranda, Some possible sources of IceCube TeV-PeV neutrino events, Eur. Phys. J. C 75, no. 6, 273 (2015).

[6] C. D. Dermer and R. Schlickeiser, Model for the high-energy emission from blazars, Astrophys. J. 416, 458 (1993).

[7] S. Sahu, L. S. Miranda and S. Rajpoot, Multi-TeV flaring from blazars: Markarian 421 as a case study, Eur. Phys. J. C 76, no. 3, 127 (2016).

[8] S. Sahu, et al., EBL effect on the observation of multi-TeV flaring of 2009 from Markarian 501 Eur. Phys. J. C 77, no. 1, 18 (2017).

[9] J. Braun, et al., Methods for point source analysis in high energy neutrino telescopes, Astropart. Phys. 29, 299 (2008). 
[10] J. A. Aguilar [IceCube Collaboration], Neutrino searches with the IceCube telescope, Nucl. Phys. Proc. Suppl. 237-238, 250 (2013).

[11] R. Enberg, M. H. Reno and I. Sarcevic, High energy neutrinos from charm in astrophysical sources, Phys. Rev. D 79, 053006 (2009). 